\title{
DYNAMIC TRAFFIC RE-ROUTING AS A METHOD OF REDUCING THE CONGESTION LEVEL OF ROAD NETWORK ELEMENTS
}

\author{
Novikov Aleksandr*1 Zyryanov Vladimir ${ }^{2}$ Feofilova Anastasiya ${ }^{2}$ \\ ${ }^{1}$ Orel State University, Russian Federation \\ ${ }^{2}$ Don State Technical University, Russian Federation
}

The article presents the main points of the dynamic traffic re-routing on the base of the Intelligent Transport System (ITS). Re-routing problem on the road network has been object many research and different tools have been proposed to increase the traffic management systems effectiveness. In this paper we study some of the issues associated with the simulation of various dynamic route guidance algorithms, focusing on the estimation of their effective using set of traffic parameters. It was founding the main condition for using the method of dynamic traffic rerouting is not only the fact or high probability of occurrence of a traffic incident, but the detection of a process of deterioration of traffic conditions, leading to the formation of congestion in the lack or ineffectiveness of active control. Our research aimed at determination the congestion threshold, alternative routes, average number of re-routing vehicles and duration of re-routing. Also in this paper is presented and explained the most important part of the dynamic traffic re-routing problem - the conceptual diagram of the main stages of the dynamic traffic re-routing.

Key words: Dynamic traffic re-routing, Traffic simulation, Alternative route, Intelligent transport system

\section{INTRODUCTION}

The efficient use of the transport city infrastructure is mostly determined by the existence and operation of intelligent transport systems (ITS). The analysis of the current traffic conditions and congestion level of main highways reveals the topical character of scientific research in the field of efficient traffic flow management. That is why the dynamic traffic re-routing from road sections with heavy traffic to the alternative routes is so important. The development of monitoring traffic flow systems and methods of traffic assignment on the road network using simulation model allows us to analyze the problem of dynamic traffic re-routing on a new level. In Russian cities with a level of motorization $300 \div 500$ veh per 1000 inhabitants, at least $20 \%$ of the sections of the main road network operate under an unacceptable v/c ratio that equal more than 0.7 . At $40 \%$ of the road network can occur synchronized traffic flow with the v/c ratio $0.45 \div 0.7$, a partially synchronized traffic flow is detected at $30-35 \%$ of the main road network, free flow conditions are almost not observed. In the presented situation, the method of reducing the traffic congestion by means of dynamic traffic re-routing (DTR) to alternative routes becomes especially topical.

\section{MATERIALS AND METHODS}

Dynamic traffic re-routing as an element of organization and management of heavy traffic is a part of ATMS and ITS. It is implemented in indirect and direct traffic flow management systems with the purpose of redirecting traffic flows from the sections with heavy traffic to alternative routes.
Our studies on Rostov on Don network have shown that redirection of $30 \%$ of the volume of traffic flows will cause a maximum (by $18 \%$ ) decrease in the proportion of road network section with an unacceptable v/c ratio. According to the data in Table 1, redirection of a greater volume of traffic flow will cause alternative routes and a reverse increase in the proportion sections of the main road network with an unacceptable v/c ratio.

Most of the research is devoted to find an alternative path, the influence of traffic information on road users, the analysis of the reactive strategies results for operational traffic flows management $[08,11,12,14]$. However, the tasks of dynamic traffic flow re-routing in case of incidents and congestions are still topical. Thus, the definition and formalization of such traffic conditions under which their dynamic re-routing is efficient are of particular significance and has not yet been studied.

Table 1: Change in performance evaluation indicators sections of the main road network with DTR

\begin{tabular}{|c|c|c|}
\hline DTR, \% & $\begin{array}{c}\text { Proportion sections } \\
\text { of the main road network } \\
\text { with an unacceptable } \\
\text { v/c ratio, \% }\end{array}$ & $\begin{array}{c}\text { Average v/c } \\
\text { ratio of the main } \\
\text { road network }\end{array}$ \\
\hline 0 & 20 & 0,53 \\
\hline 10 & 17 & 0,52 \\
\hline 20 & 9 & 0,49 \\
\hline 30 & 4 & 0,49 \\
\hline 40 & 8 & 0,49 \\
\hline
\end{tabular}


For many years the study of the relationship between the main characteristics of traffic flow is one of the main directions of theoretical and experimental research in the theory of traffic flow. The contradiction between the real and calculated data in the basic models and the choice of appropriate models are studied by J. Castillo (1995), F. Benitez, R. Herman, I. Prigogin, V. Zyryanov (2008). Research and prediction of traffic transition from the free state are reflected in the alternative theory of B. Kerner's (2009) traffic flow. Many traffic simulations using AIMSUN software showed the same type of traffic flow phase diagrams in case of congestions on the study route regardless of their origin. [07, 09, 11, 13]

Traffic flow phases diagram provides the presented characteristics of traffic flow: flow $q_{i}$, density at $k_{i}$. More accurate studying reveals $k_{\text {thresh }}$ as the traffic flow density at which there is a transition from free flow phase $(F)$ to synchronized flow phase (S). This indicates a change in traffic conditions for the worse and causes congestion if there is a lack of traffic management. These fundamental dependencies presented in Figure 1 allowed to use $k_{\text {thresh }}$ as a reason for dynamic traffic flows re-routing. Volume at $k_{\text {thresh }}$ is the width of the hysteresis loop which describes a transition $F \rightarrow J$ (traffic jam phase) and the reverse re-routing $J \rightarrow F$. We define the width as $\Delta q=q_{2}\left(k_{\text {thresh }}\right)-q_{1}\left(k_{\text {thresh }}\right)=Q_{\text {effect }}$. Figure 1 graphically presents a methodology for determining the threshold parameters of the traffic flow and a part of re-routing vehicles. It also depicts the sustainability of the alternative path that takes the effective volume of re-routing vehicles. Let the alternative path detect traffic flow condition which can be characterized as $(\bullet) B\left(k_{i} ; q_{i}\right)$. If you are re-routing to an alternative vehicle path $Q_{\text {effect }}$ we will get a new traffic flow condition which can be characterized as $(\bullet) \hat{A}_{\text {new }}\left(k_{j} ; q_{i}+Q_{\text {effect }}\right)$ and meets the free traffic conditions. Consequently, the re-routing $Q_{\text {effect }}$ will not cause rejection of alternative path. And dynamic re-routing can be extended as long until $(\bullet) A_{\text {new }}\left(k_{n} ; q_{\text {thresh }}-Q_{\text {effect }}\right)$ is reached (Figure 1).

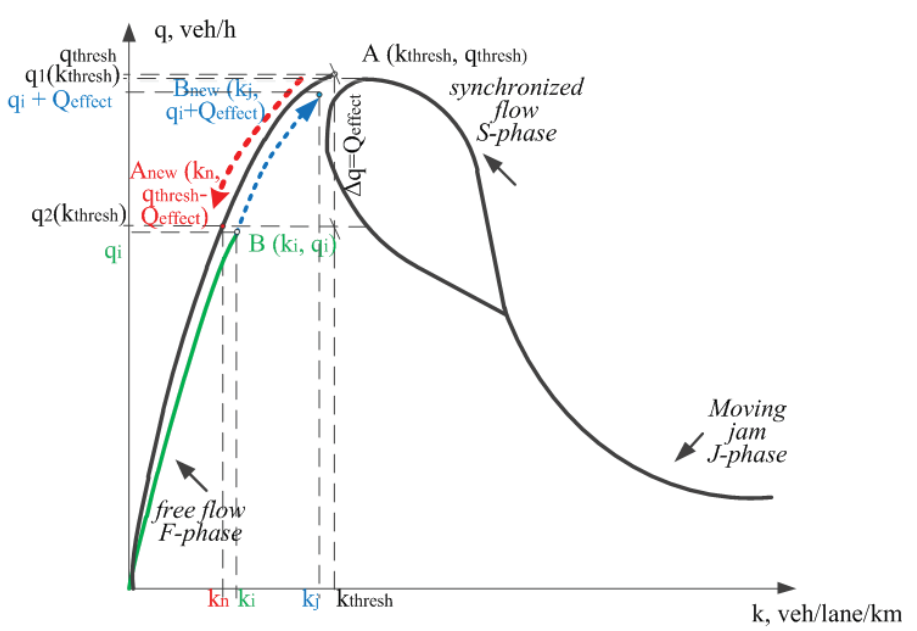

Figure 1: Determination schematic for use condition for dynamic traffic flow re-routing
At the same time, the implementation of DTR is largely determined by the need to ensure the sustainability of those sections of the road network, which are a part of the recommended alternative route. Therefore, the end of the re-routing of traffic flow to an alternative route should occur after reaching the limit values that characterize the traffic conditions, the definition of which is the criterion for introducing this method of organizing traffic. The diagrams have shown on Figure 2 allows to define the point of sections sustainability based on known characteristic of traffic flow which can be detected: flow, density and speed.

\section{RESULTS}

Determination of the place, role and special requirements for the implementation of this method of traffic management is possible by means of the construction of a physical and functional architecture for the project of DTR. At the same time the application of this method is determined to a certain extent by the architecture of the city's ITS creation, the functioning and development of its integrated and instrumental subsystems, the meeting of the requirements for their effective deployment based on traffic simulation.

The conceptual diagram of the main stages of the DTR is presented in Figure 3.

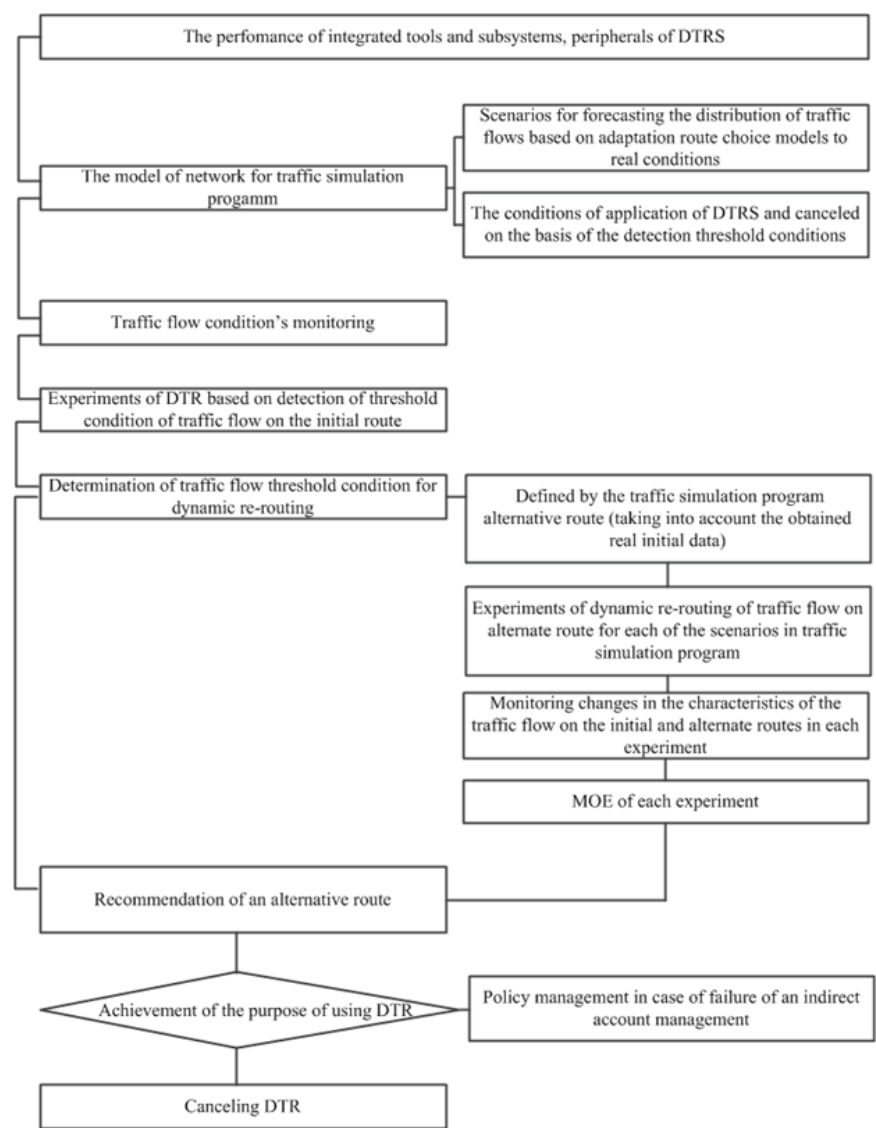

Figure 3: Conceptual diagram of the main stages of the DTR 


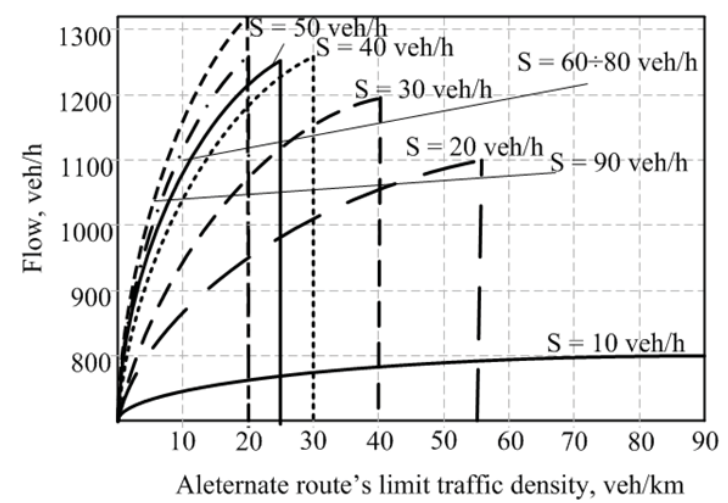

a)

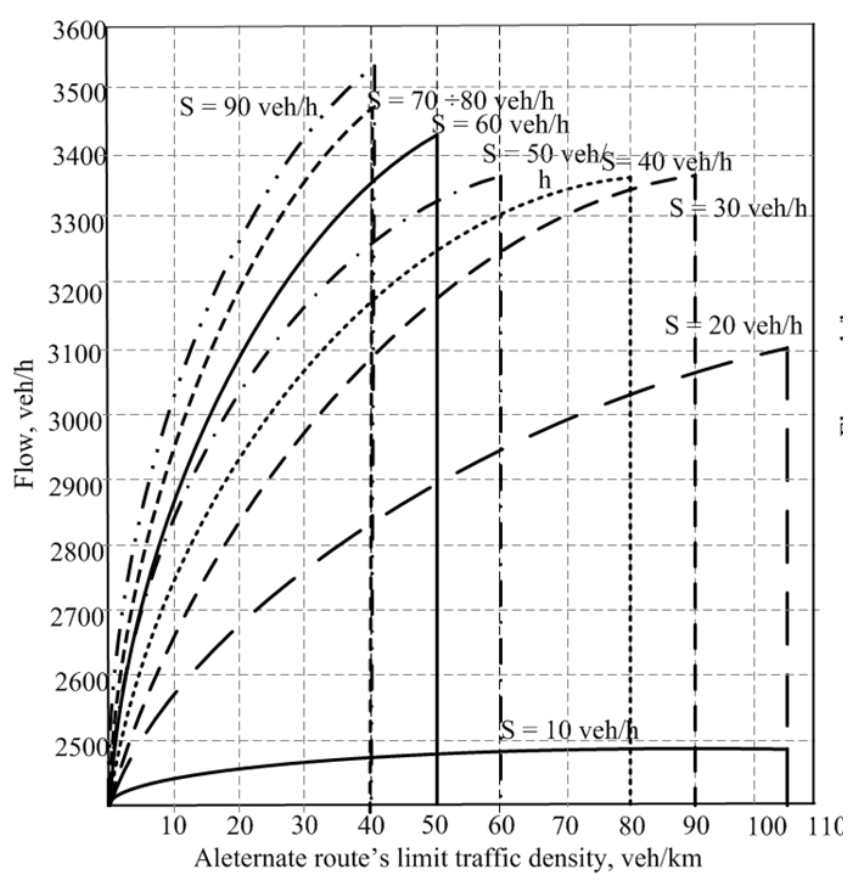

c)

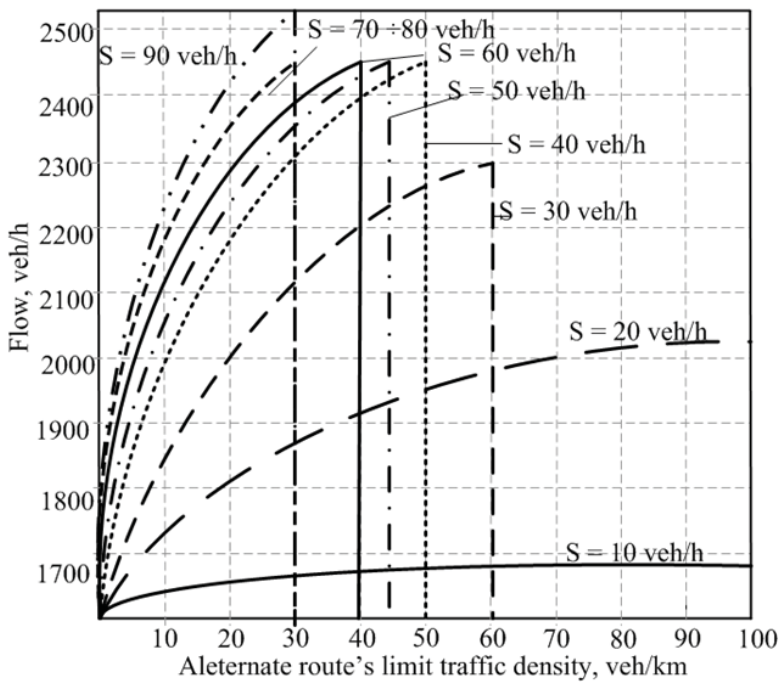

b)

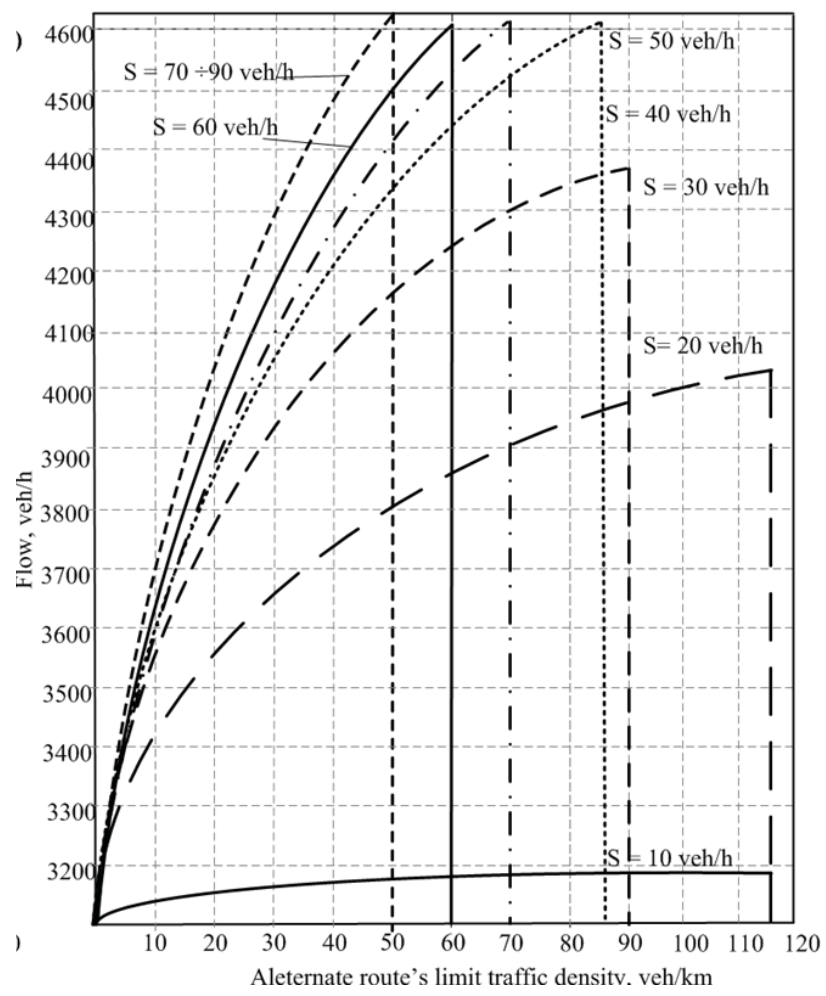

d)

a) one lane on the route direction

b) two lane on the route direction

c) three lane on the route direction

d) four lane on the route direction

Figure 2: Diagram for determination of the sections sustainability 


\section{THE MAIN POINTS OF THE DYNAMIC TRAFFIC RE-ROUTING}

The main condition for using the method of dynamic traffic rerouting is not only the fact or high probability of occurrence of a traffic incident, but the detection of a process of deterioration of traffic conditions, leading to the formation of congestion in the lack or ineffectiveness of active control. The undertaken studies showed that the use of DTR in the initial conditions of motion, determined, for example, by the level of service $A$, would be efficient only in $2 \%$ of the traffic situations determined by traffic flow threshold condition. Whereas, in the initial traffic conditions, determined, for example, by the level of service $E$, the method of DTR is effective in $71 \%$ of cases (see Figure 4).

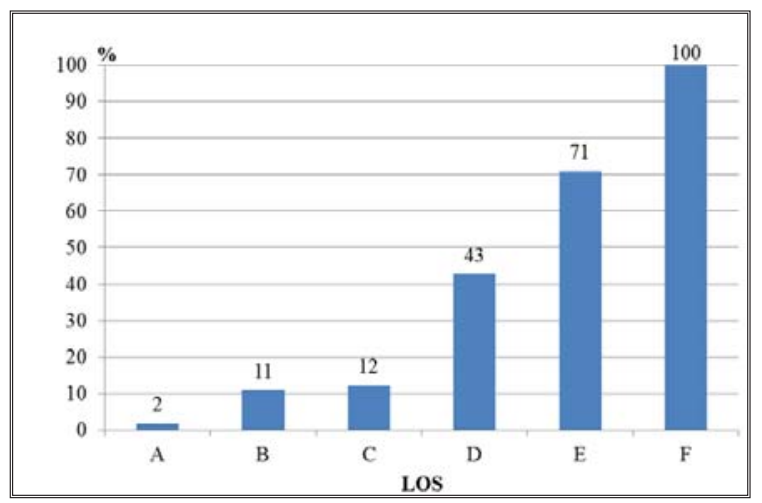

Figure 4: Distribution in the initial traffic conditions that require their dynamic rerouting at the initial service levels

Implementing the dynamic traffic flow re-routing for sustainable traffic of road network sections as a part of recommended alternative route it is necessary to obtain initial data on road traffic and traffic flow conditions. It enables the process of dynamic re-routing in order to avoid congestion.

The next key issue is the determination of traffic flow threshold condition to start the dynamic rerouting, depending on the change in the initial traffic conditions in the research road network section. Previous studies have shown that the factors affecting the change of traffic condition's threshold are a number of section lanes and section free flow speed.

With the help of simulation, we defined the area of efficient application of the considered method of traffic organization with the change of the initial $\mathrm{v} / \mathrm{c}$ ratio on the road sections. [14, 15]. We obtained the diagrams for determining the threshold density of the traffic flow under conditions of varying level of services (LOS) in the direction of the traffic flow along the route. It allows to answer one of the most important questions about the beginning of dynamic traffic rerouting (see Figure 5).

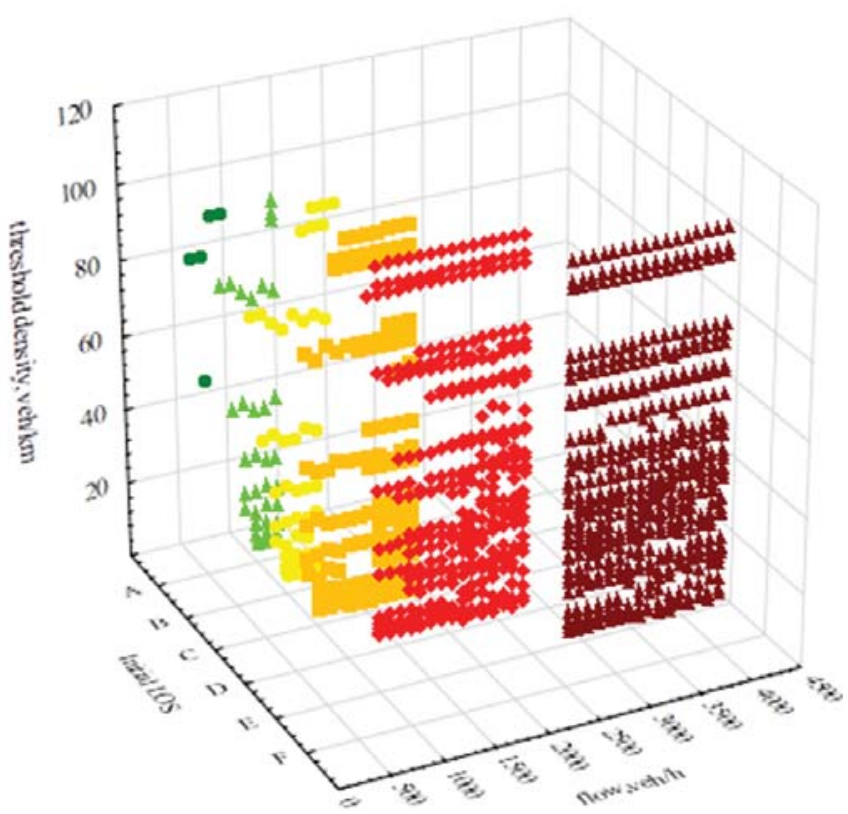

Figure 5: Diagram for determination of traffic flow threshold condition for dynamic re-routing

Achievement of the goals to prevent the occurrence and reduction of the duration of the road congestion, to avoid traffic breakdown it is important to choose the detection area for the threshold states of traffic flows.

The condition for the ending the dynamic rerouting is measure the characteristics of the traffic flow on the initial route, which determine the transition of traffic to the free state allowed by the capacity values.

The efficiency of DTR is determined by the reduction of time and losses from road accidents, the increased reliability of road network operation, the reduction of transportation costs and pollutant emissions.

\section{DISCUSSION}

According to the research carried out by the authors, the reduction in the travel time during the congestion with the use of DTR can reach half as much. It indicates the high efficiency of this method in terms of reducing the duration of the congestion [14, 15].

The paper presents research aimed at finding the conditions for the start of the dynamic traffic flows re-routing as a point of traffic flow density at which there is a transition to synchronized flow phase. We calculate the conditions for traffic rerouting determined by the network parameters and the speed limits. We found out traffic flow conditions at which their dynamic re- routing is efficient. Our proposed method of evaluating the conditions for the starting point of traffic re-routing can also determine its efficiency. At the same time we can also evaluate the conditions on the alternate route: they can be stable or we need to find additional routes to reduce the congestion on the original route. Applying space-time diagrams allow us to track the change in traffic states on the route while performing any impact. 
Further research has the following tasks: to predict the traffic situation in the development of transport infrastructure during process of transport planning; to study the traffic flows' redistribution on the new network; to forecast the traffic situation in the case of incidents; to improve methods and algorithms for the dynamic traffic re-routing.

\section{CONCLUSIONS}

Application of DTR is determined to a certain extent by the architecture of the city's ITS creation, the functioning and development of its integrated and instrumental subsystems, the meeting of the requirements for their effective deployment based on traffic simulation. Re-routing problem on the road network has been object many research and different tools have been proposed to increase the traffic management systems effectiveness.

The first of most important part of the dynamic traffic re-routing problem - the conceptual diagram of the main stages of the dynamic traffic re-routing. We presented and explained each stage of DTR on our paper.

The originality part of our research is the distribution in the initial traffic conditions that require their dynamic rerouting at the initial service levels. Also we found the diagram for determination of the sections sustainability.

The most important and newness result of our research is the diagrams for determination of traffic flow threshold condition for dynamic re-routing. .It allows to answer one of the most important questions about the beginning of dynamic traffic rerouting.

\section{REFERENCES}

1. Daganzo, C.F., Geroliminis, N. (2008), "An analytical approximation for the macroscopic fundamental diagram of urban traffic", Transportation Research Part B 42, Pages 771-781

2. Del Castilio, J.M., Benitez, F.G. On the functional form of the speed-density relationship - I: General theory. "Transp. Res.". 1995, vol.29B, №5. p.p. 373389

3. Del Castilio, J.M., Benitez, F.G. On the functional form of the speed-density relationship - II:Empirical investigation. "Transp. Res.". 1995, vol.29B, №5. p.p. 391-406

4. Florian, M., Mahut, M., Tremblay, N. (2008). Application of a simulation-based dynamic traffic assignment model, European Journal of Operational Research. 189 (3) (2008) 1381-1392.

5. Fundamentals of Traffic Simulation. Ed. Barcelo, J. (2010). Springer. 440 p.

6. Geroliminis, N., Daganzo, C.F. (2008), "Existence of urban-scale macroscopic fundamental diagrams: Some experimental findings", Transportation Research Part B 42, Pages 759-770.
7. Kerner, B. S. (2009). Introduction to Modern Traffic Flow Theory and Control: The Long Road to ThreePhase Traffic Theory. The Long Road to ThreePhase Traffic Theory, 265p

8. Knoop V. L., Hoogendoorn S.P., Van Lint J.W.C. (2011) Routing Strategies based on the Macroscopic Fundamental Diagram. Transportation Research Record: Journal of the Transportation Research Board. Issue Number: 2315, 2012, pp 1-10

9. Mahmassani, H.S., Williams, J., Herman, R. (1987), "Performance of urban traffic networks", Proceedings of the 10th International Symposium on Transportation and Traffic Theory, pp. 1-20

10. Rao, A. M., Rao, K. (2012). Measuring urban traffic congestion - a review. International Journal for Traffic and Transport Engineering, 2012, 2(4) pp. $286-305$

11. Sheffi, Y., (1985). Urban Transportation Networks: Equilibrium Analysis with Mathematical Programming Methods. Prentice-Hall, Englewood Cliffs, USA.

12. Sundaram, S. et al. (2010). Simulation-based dynamic traffic assignment for short-term planning applications, Simulat. Modell. Pract. Theory (2010), doi:10.1016/j.simpat.2010.08.004

13. Zyryanov V. (2008). Simulation of Impact of Components of ITS on Congested Traffic States. Proceedings of 7 th European Congress on Intelligent Transport Systems. Geneva. 2008. ID 2694. p. 1-5

14. Zyryanov Vladimir, Feofilova Anastasia. Evaluation Parameters of Re-routing Strategy. Traffic Management: DOI: 10.1002/9781119307822.ch14 , pp.203216

15. Vladimir Zyryanov, Anastasia Feofilova. Simulation of Evacuation Route Choice. Transportation Research Procedia Volume 20, 2017, Pages 740-745

Paper submitted: 20.10.2017.

Paper accepted: 08.02.2018.

This is an open access article distributed under the CC BY-NC-ND 4.0 terms and conditions. 